\title{
Poster: Recent Results in State Estimation of Dynamical Systems with Inputs under Bandwidth Constraints
}

\author{
Hussein Sibai and Sayan Mitra \\ \{sibai2,mitras\}@illinois.edu \\ University of Illinois at Urbana-Champaign \\ Coordinated Science Laboratory \\ Urbana, IL 61801
}

\begin{abstract}
Finding the minimal bit rate needed for state estimation of a dynamical system is a fundamental problem in control theory. We present two notions of topological entropy, one to lower bound the bit rate needed to estimate the state of a nonlinear dynamical system, with unknown bounded inputs, up to a constant error $\varepsilon$. The other is to do the same but to estimate the state of a switched system with unknown switching signal up to an error that is bounded by $\varepsilon$ for $\tau$ seconds after each switch and then decays exponentially at a rate of $\alpha$ till the next switch. Since computation of entropy is hard in general, we present upper bounds on both notions of entropy. Finally, we present preliminary results on the relation between the two notions. Note that most of the ideas presented in this abstract are from our papers [4] and [5].
\end{abstract}

\section{ACM Reference Format:}

Hussein Sibai and Sayan Mitra. 2018. Poster: Recent Results in State Estimation of Dynamical Systems with Inputs under Bandwidth Constraints. In HSCC '18: 21st International Conference on Hybrid Systems: Computation and Control (part of CPS Week), April 11-13, 2018, Porto, Portugal. ACM, New York, NY, USA, 2 pages. https://doi.org/10.1145/3178126.3187002

\section{INTRODUCTION}

State estimation is a fundamental problem for controlling and monitoring dynamical systems. In most application scenarios, the estimator has to work with plant state information sent by a sensor over a channel which permits only finite bit rates. If a certain level of accuracy is required from the estimator, then a natural question is to ask: what is the minimal bit rate needed for the channel to support this requirement? This question has been investigated for both stochastic and non-stochastic systems and channels. In the non-stochastic setting, the point of view of topological entropy has proved to be fruitful line of investigation for deriving minimal necessary bitrates for systems without inputs (see, for example [2-4]). From the point of view of estimation, the topological entropy of a dynamical system represents the rate at which the number of distinguishable trajectories with a predefined precision exponentially increase over

We thank Daniel Liberzon for providing detailed and insightful comments. This work is in part supported by research grants AFOSR FA9550-17-1-0236 and NSF 1739966.

Permission to make digital or hard copies of part or all of this work for personal or classroom use is granted without fee provided that copies are not made or distributed for profit or commercial advantage and that copies bear this notice and the full citation on the first page. Copyrights for third-party components of this work must be honored.

For all other uses, contact the owner/author(s).

HSCC '18, April 11-13, 2018, Porto, Portugal

(C) 2018 Copyright held by the owner/author(s).

ACM ISBN 978-1-4503-5642-8/18/04.

https://doi.org/10.1145/3178126.3187002 time. This poster contributes in that line of research and proposes answers for systems with bounded, piece-wise continuous input of the form:

$$
\dot{x}(t)=f(x(t), u(t)),
$$

with $f$ being globally Lipschitz continuous in both arguments, in addition to switched systems of the form:

$$
\dot{x}(t)=f_{\sigma(t)}(x(t))
$$

with $f_{i}, i \in\{1, \ldots, N\}$, being globally Lipschitz continuous and $\sigma$ a piecewise-constant function mapping time to one of the $N$ possible modes with known minimal dwell time. Estimating the states of systems with inputs or those of switched systems are challenging problems because even if the uncertainty about the state can be made to decrease over time using sensor measurements, the uncertainty about the input or mode may not decrease. The input can change arbitrarily with few constraints and the continuous effect of the uncertain input prevents the uncertainty about the state from going to zero. Similarly, the switching signal may switch between different modes with the dwell time being the only constraint. We contend this using a weaker notion of estimation, that only requires the error to be bounded by a constant $\varepsilon>0$ instead of exponentially decaying to zero for systems with inputs and to require it to decay exponentially in the case of switched systems but only after a specified period of time after each switch. Hence, we define the topological entropy for system (1) as follows:

$$
h_{\mathrm{u}, \mathrm{est}}(\varepsilon, K)=\limsup _{T \rightarrow \infty} \frac{1}{T} \log s_{\mathrm{u}, \mathrm{est}}(T, \varepsilon, K),
$$

and that of system (2) by:

$$
h_{\mathrm{s}, \mathrm{est}}(\varepsilon, \alpha, \tau, K):=\limsup _{T \rightarrow \infty} \frac{1}{T} \log s_{\mathrm{s}, \mathrm{est}}(T, \varepsilon, \alpha, \tau, K),
$$

where $K$ is the compact set of possible initial states, $\varepsilon$ is the allowed estimation error for system (1) and $s_{\mathrm{u}, \mathrm{est}}(T, \varepsilon, K)$ is the minimal cardinality of a function set (from time to states) that for every trajectory of system (1) over the time interval $[0, T]$ originating from $K$ has a function that approximates it up to an $\varepsilon$ error. Moreover, $\varepsilon, \tau$ and $\alpha$ are parameters that specify the allowed estimation error for system (2). $s_{\mathrm{s} \text {,est }}$ is the minimal cardinality of a set of functions over a time interval $[0, T]$ where for each $x_{0} \in K$ and each $\sigma$ (with minimal dwell time), there exists a $z \in s_{\mathrm{s}, \text { est }}$, where for all $j \geq 0$ and for all $t \in\left[s_{j}, s_{j+1}\right)$,

$$
\left|z(t)-\xi_{\sigma}\left(x_{0}, t\right)\right| \leq \begin{cases}\varepsilon & t \in\left[s_{j}, s_{j}+\tau\right), \\ \varepsilon e^{-\alpha\left(t-\left(s_{j}+\tau\right)\right)} & \text { otherwise, }\end{cases}
$$


where $s_{0}=0, s_{1}, \ldots$ are the switching times in $\sigma$ and $\xi_{\sigma}\left(x_{0}, \cdot\right)$ is the trajectory of system (2).

Relation between Entropy and Bit Rates of State Estimation Algorithms. We proved in both papers that there is no state estimation algorithms with a fixed bit rate, defined as

$$
b_{r}(\varepsilon, K):=\limsup _{T \rightarrow \infty} \frac{1}{T} \sum_{i=0}^{\left\lfloor T / T_{p}\right\rfloor} \log N=\frac{\log N}{T_{p}},
$$

for system (1) and as

$$
b_{r}(\varepsilon, \alpha, \tau, K):=\limsup _{T \rightarrow \infty} \frac{1}{T} \sum_{i=0}^{\left\lfloor T / T_{p}\right\rfloor} \log N=\frac{\log N}{T_{p}},
$$

where $\log N$ is the number of bits sent each $T_{p}$ seconds by the sensor to the estimator, $K$ is a compact set of possible initial states, and $\varepsilon, \alpha$ and $\tau$ are the parameters that specify the allowed error, that are smaller than $h_{\mathrm{u} \text {,est }}$ and $h_{\mathrm{s} \text {,est }}$.

\section{ENTROPY UPPER BOUND FOR SYSTEM (1)}

Computing entropy is hard in general, so we resort to computing bounds on it. For the purpose of computing an upper bound, we use a corrected version of a previous result in [1] to upper bound the sensitivity of a trajectory of a nonlinear system to changes in the initial state and in the input signal. Then, we construct an algorithm that, given sampled states of a trajectory and corresponding sampled values of an input signal, constructs a function that estimates the trajectory up to an $\varepsilon$ error. This procedure is of independent interest, as it can be used as an estimation algorithm if the unknown input signal can be sampled. We count the number of trajectories that can be constructed by this procedure for different initial states and input signals, up to a time bound $T$. The rate of growth of this number gives an upper bound on entropy.

The upper bound is presented in terms of the state and the input dimensions $n$ and $m$, global bounds on the norm of the Jacobian matrices of the vector field with respect to the state and the input, $M_{x}$ and $M_{u}$, the upper bound on the norm of the input $u_{\max }$, and two constants $\mu$ and $\eta$ that represent how much the input signal is allowed to vary over time. Roughly, $\eta$ upper bounds the size of the jumps in the input signal and $\mu$ constrains the number of large jumps in a short amount of time. More formally, $\mu$ and $\eta$ should satisfy $\|u(t+\tau)-u(t)\| \leq \mu \tau+\eta$, for all $t$ and $\tau \geq 0$ and any possible input signal $u$.

Proposition 2.1. Fix $\varepsilon>0$ and $a$ constant $k \in(0,1)$. Then, choose $T_{p}$ and $\delta_{u}>0$, such that:

$$
\varepsilon \sqrt{1-k} \geq M_{u} e^{M_{x} T_{p}} \sqrt{\frac{1}{3} \mu^{2} T_{p}{ }^{3}+\left(\delta_{u}+\eta\right) \mu T_{p}{ }^{2}+\left(\delta_{u}+\eta\right)^{2} T_{p}},
$$

and let

$$
\rho\left(k, \delta_{u}\right)=\left(\frac{\delta_{u}+\eta}{\mu}\right)\left(-1+\sqrt[3]{1+\left(\frac{\varepsilon}{M_{u} e}\right)^{2} \frac{3 \mu(1-k)}{\left(\delta_{u}+\eta\right)^{3}}}\right) .
$$

Then, the entropy of system (1) is upper bounded by:

$$
\frac{n M_{x}}{\sqrt{k} \ln 2}+\frac{1}{\min \left\{\rho\left(k, \delta_{u}\right), 1 / M_{x}\right\}}\left(n \log (1+\sqrt{k})+m \log \left\lceil\frac{u_{\text {max }}}{\delta_{u}}\right\rceil\right) .
$$

For example, $k$ and $\delta_{u}$ can be $1 / 2$ and $\eta$, respectively.
We showed in the paper that if the upper bound on the input norm goes to zero, we recover, up to $O(n)$ factor, the upper bound on estimation entropy $\frac{n L_{x}}{\ln 2}$ computed in [2] for $\alpha$ equal to zero. The entropy upper bound increases logarithmically with $u_{\max }$, and quadratically with $\eta$ when $\varepsilon$ is small. It also increases as $O\left(\varepsilon^{-2}\right)$ as the allowed estimation error $\varepsilon$ decreases.

\section{ENTROPY UPPER BOUND FOR SYSTEM (2)}

In [4], we presented a similar result to that in [5] but for switched nonlinear systems with unknown switching signals with known dwell time $T_{d}$. We presented our notion of topological entropy for state estimation of such systems over limited-bandwidth channels. We computed an upper bound on entropy and presented a state estimation algorithm when the switching signal is not observable.

To compute an upper bound on entropy, we assumed that a bound on the distance between the states resulting from running two different modes from the same initial state exists for all $t \leq \tau$ :

$$
d(t):=\max _{p, r \in[N]} \sup _{x \in \operatorname{Reach}(\Sigma, K)} \int_{0}^{t}\left\|f_{p}\left(\xi_{p}(x, s)\right)-f_{r}\left(\xi_{r}(x, s)\right)\right\| d s,
$$

which is true, for example, if the reach set is compact. We used a similar method to that in the previous section to compute the upper bound. It is presented in the following proposition.

Proposition 3.1. Let $T_{e}$ be the largest in $(0, \tau]$ that satisfies $d\left(T_{e}\right) \leq \varepsilon\left(1-e^{-\alpha\left(T_{d}-T_{e}\right)}\right)$. Then, the entropy $h_{e s t}(\varepsilon, \alpha, \tau) \leq(L+$ $\alpha) n / \ln 2+(\log N) / T_{e}$.

Relation between the two notions of entropy. Note that if one requires the estimation error to be upper bounded by a constant in switched systems, she can use the results in the previous section to get an upper bound on entropy. To do that, she should model the different modes of the system as different inputs to the nonautonomous system. Then, she should bound the difference between the dynamics of the different modes to get an upper bound on the Jacobian matrix of $f$ with respect to the input in system (1). Finally, she needs to know the dwell time of the system to get the values of $\mu$ and $\eta$. This will be formalized in future work.

Limit supremum as $\varepsilon$ goes to zero. Note that in our definition of entropy we do not take the limit supremum as $\varepsilon$ goes to zero which is the case in the definition of topological entropy. In fact, as the allowed estimation error $\varepsilon$ goes to zero, not only the upper bounds on entropy presented in Propositions 2.1 and 3.1 will diverge to infinity, but also both entropy values would also diverge. We have a priliminary proof that will be presented in a future work.

\section{REFERENCES}

[1] Zhenqi Huang, Chuchu Fan, and Sayan Mitra. 2017. Bounded invariant verification for time-delayed nonlinear networked dynamical systems. Nonlinear Analysis: Hybrid Systems 23 (2017), 211 - 229. https://doi.org/10.1016/j.nahs.2016.05.005

[2] Daniel Liberzon and Sayan Mitra. 2016. Entropy and minimal data rates for state estimation and model detections. In HSCC 2016. ACM, Vienna.

[3] Daniel Liberzon and Sayan Mitra. 2017. Entropy and minimal bit rates for state estimation and model detection. In IEEE Transactions in Automatic Control. IEEE.

[4] Hussein Sibai and Sayan Mitra. 2017. Optimal data rate for state estimation of switched nonlinear systems. Association for Computing Machinery, Inc, 71-80. https://doi.org/10.1145/3049797.3049799

[5] Hussein Sibai and Sayan Mitra. 2018. State Estimation of Dynamical Systems with Unknown Inputs: Entropy and Bit Rates. Association for Computing Machinery, Inc. https://doi.org/10.1145/3178126.3178150 